\title{
Tubeless video-assisted thoracic surgery for pulmonary ground-glass nodules: expert consensus and protocol (Guangzhou)
}

Jianxing He ${ }^{1}$, Hengrui Liang ${ }^{1}$, Wei Wang ${ }^{1}$, Andrey Akopov ${ }^{2}$, Alberto Aiolfi ${ }^{3}$, Keng-Leong Ang ${ }^{4}$, Luca Bertolaccini ${ }^{5}$, Kaican Cai ${ }^{6}$, Qingdong Cao ${ }^{7}$, Baojun Chen ${ }^{8}$, Chang Chen ${ }^{9}$, Chun Chen ${ }^{10}$, Donglai Chen', Fengxia Chen $^{11}$, Jun Chen ${ }^{12}$, Lei Chen ${ }^{13}$, Mingwu Chen ${ }^{14}$, Yongbing Chen ${ }^{15}$, Zhuxing Chen ${ }^{1}$, Chao Cheng ${ }^{16}$, Dong Cui ${ }^{17}$, Fei Cui ${ }^{1}$, Tianyang Dai ${ }^{18}$, Qinglong Dong ${ }^{13}$, Paolo A. Ferrari ${ }^{19}$, Raja M. Flores ${ }^{20}$, Junke Fu ${ }^{21}$, Soichiro Funaki ${ }^{22}$, Marios E. Froudarakis ${ }^{23}$, Xiangfeng Gan ${ }^{7}$, Mingfei Geng $^{24}$, Jialong Guo ${ }^{25}$, Qiang Guo ${ }^{26}$, Yongtao $\mathrm{Han}^{27}$, Jintao $\mathrm{He}^{28}$, Kaiming $\mathrm{He}^{18}$, Kyoji $\mathrm{Hirai}^{29}$, Jian $\mathrm{Hu}^{30}$, Shuqiao $\mathrm{Hu}^{31}$, Jian Huang ${ }^{32}$, Jun Huang ${ }^{1}$, Wenfa Jiang ${ }^{33}$, Kyung Soo Kim ${ }^{34}$, Gabor Kiss ${ }^{35}$, Fanyi Kong ${ }^{36}$, Lan Lan ${ }^{13}$, Xuefeng Leng ${ }^{27}$, Bin Li ${ }^{37}$, Gaofeng Li ${ }^{38}$, Hecheng $\mathrm{Li}^{39}$, Hefei $\mathrm{Li}^{26}$, Heng $\mathrm{Li}^{38}$, Jiwei $\mathrm{Li}^{40}$, Xiaoqiang $\mathrm{Li}^{41}$, Shuben $\mathrm{Li}^{1}$, Yinfen $\mathrm{Li}^{13}$, Zhuoyi $\mathrm{Li}^{1}$, Yi Liang ${ }^{42}$, Lixia Liang ${ }^{13}$, Wenhua Liang ${ }^{1}$, Yongde Liao ${ }^{43}$, Wanli Lin ${ }^{44}, \mathrm{Xu} \mathrm{Lin}^{30}$, Hongxu Liu ${ }^{45}$, Hui Liu ${ }^{13}$, Jixian Liu ${ }^{41}$, Jun Liu ${ }^{1}$, Xiang Liu ${ }^{46}$, Zihao Liu ${ }^{13}$, Xingzhao Lu ${ }^{47}$, Qingquan Luo ${ }^{48}$, Naiquan Mao ${ }^{49}, Q$ Pan $^{49}$, Dazhi Pang $^{50}$, Jun Peng ${ }^{51}$, Jun Peng ${ }^{28}$, Eugenio Pompeo ${ }^{52}$, Rulin Qian ${ }^{17}$, Kun Qiao ${ }^{53}$, Bassam Redwan ${ }^{54}$, Zi Sang ${ }^{24}$, Wenlong Shao ${ }^{1}$, Jianfei Shen ${ }^{55}$, Weiyu Shen ${ }^{56}$, Sook-Whan Sung ${ }^{57}$, Wenfang Tang ${ }^{42}$, Tianhu Wang ${ }^{58}$, Guangsuo Wang ${ }^{59}$, Haitao Wang ${ }^{60}$, Huien Wang ${ }^{61}$, Jiyong Wang ${ }^{62}$, Wen Wang ${ }^{63}$, Yongyong Wang ${ }^{15}$, Zhenyuan Wang ${ }^{64}$, Li Wei ${ }^{40}$, Wei Wei ${ }^{65}$, Hao Wu ${ }^{66}$, Jie Wu ${ }^{67}$, Zhaohua Xia ${ }^{53}$, Chenyang Xu ${ }^{33}$, Enwu Xu ${ }^{68}$, Hai $\mathrm{Xu}^{69}$, Ning $\mathrm{Xu}^{70}$, Quan Xu ${ }^{66}$, Rongyu Xu ${ }^{71}$, Shun $\mathrm{Xu}^{72}$, Chaokun Yang ${ }^{73}$, Hanyu Yang ${ }^{13}$, Shengli Yang ${ }^{74}$, Jun Yi $^{75}$, Guangjian Zhang ${ }^{21}$, Hao Zhang ${ }^{76}$, Jia Zhang ${ }^{21}$, Man Zhang ${ }^{77}$, Xiao Zhang ${ }^{78}$, Yajie Zhang ${ }^{39}$, Zhe Zhang $^{79}$, Zhifeng Zhang ${ }^{80}$, Honglin Zhao ${ }^{13}$, Jian Zhao ${ }^{81}$, Xiaodong Zhao ${ }^{82}$, Jianping Zhou ${ }^{47}$, Yanran Zhou ${ }^{13}$, Chengchu Zhu ${ }^{55}$, Shaojin Zhu ${ }^{83}$, Xinhai Zhu ${ }^{84}$, Jian Cui ${ }^{85}$, Yubo Yan ${ }^{69}$, Ke-Neng Chen ${ }^{86}$

${ }^{1}$ Department of Thoracic Surgery and Oncology, the First Affiliated Hospital of Guangzhou Medical University, State Key Laboratory of Respiratory Disease, National Clinical Research Center for Respiratory Disease, Guangzhou Institute of Respiratory Health, Guangzhou, China; ${ }^{2}$ Department of Thoracic Surgery, First Pavlov State Medical University, Saint-Petersburg, Russia; ${ }^{3}$ Division of Minimally Invasive Surgery, Istituto Clinico Sant'Ambrogio, University of Milan, Milan, Italy; ${ }^{4}$ Department of Thoracic Surgery, Glenfield Hospital, Leicester, UK; ${ }^{5}$ Department of Thoracic Surgery, IEO European Institute of Oncology IRCCS, Milan, Italy; ${ }^{6}$ Department of Thoracic Surgery, Nanfang Hospital of Southern Medical University, Guangzhou, China; ${ }^{7}$ Department of Thoracic Surgery, the Fifth Affiliated Hospital of Sun Yat-Sen University, Zhuhai, China; ${ }^{8}$ Department of Thoracic Surgery, The Central Hospital of Wuhan, Tongji Medical College, Huazhong University of Science and Technology, Wuhan, China; 'Department of Thoracic Surgery, Shanghai Pulmonary Hospital, Tongji University School of Medicine, Shanghai, China; ${ }^{10}$ Department of Thoracic Surgery, Fujian Medical University Union Hospital, Fuzhou, China; ${ }^{11}$ Department of Thoracic Surgery, Hainan General Hospital, Hainan, China; ${ }^{12}$ Lung Cancer Department, Tianjin General Hospital, Tianjin Medical University, Tianjin, China; ${ }^{13}$ Department of Anesthesia, the First Affiliated Hospital of Guangzhou Medical University, Guangzhou, China; ${ }^{14}$ Department of Cardiothoracic Surgery, the First Affiliated Hospital of Guangxi Medical University, Nanning, China; ${ }^{15}$ Department of Thoracic Surgery, the Second Affiliated Hospital of Soochow University, Soochow, China; ${ }^{16}$ Department of Thoracic Surgery, First Affiliated Hospital of Sun Yat-sen University, Guangzhou, China; ${ }^{17}$ Department of Thoracic Surgery, Henan Provincial Chest Hospital, Zhengzhou, China; ${ }^{18}$ Department of Thoracic Surgery, Southwest Medical University Affiliated Hospital, Luzhou, China; ${ }^{19}$ Division of Thoracic Surgery, Oncology Hospital “A. Businco”, A.R.N.A.S. “G. Brotzu”, Cagliari, Italy; ${ }^{20}$ Department of Thoracic Surgery, Mount Sinai Health System, Icahn School of Medicine at Mount Sinai, New York, NY, USA; ${ }^{21}$ Department of Thoracic Surgery, the First Affiliated Hospital of Xi'an Jiaotong University, Xi'an, China; ${ }^{22}$ Department of General Thoracic Surgery, Osaka University Graduate School of Medicine, Osaka, Japan; ${ }^{23}$ Department of Pneumonology and Thoracic Oncology, North Hospital, University Hospital of Saint-Etienne, St-Etienne, France; ${ }^{24}$ Department of Thoracic Surgery, Anyang Tumour Hospital, Anyang, China; ${ }^{25}$ Department of Cardiothoracic Surgery, Taihe Hospital, Hubei University of Medicine, Shiyan, China; ${ }^{26}$ Department of Thoracic Surgery, Affiliated Hospital of Hebei University, Baoding, China; ${ }^{27}$ Division of Thoracic Surgery, Sichuan Cancer Hospital \& Institute, School of Medicine, University of Electronic Science and Technology of China (UESTC), Chengdu, China; ${ }^{28}$ Department of Thoracic Surgery, Sichuan Cancer Hospital \& Institute, Sichuan Cancer Center, School of Medicine, University of Electronic Science and Technology of China, Chengdu, China; ${ }^{29}$ Division of Thoracic 
Surgery, Nippon Medical School Chiba Hokusoh Hospital, Chiba, Japan; ${ }^{30}$ Department of Thoracic Surgery, The First Affiliated Hospital, School of Medicine, Zhejiang University, Hangzhou, China; ${ }^{31}$ Department of Thoracic Surgery, Longyan City First Hospital, Longyan, China; ${ }^{32}$ Department of Thoracic Surgery, Maoming People's Hospital, Maoming, China; ${ }^{33}$ Department of Thoracic Surgery, Ganzhou People's Hospital, Ganzhou, China; ${ }^{34}$ Department of Thoracic and Cardiovascular Surgery, Seoul St. Mary's Hospital, College of Medicine, The Catholic University of Korea, Seoul, Korea; ${ }^{35}$ Department of Cardiovascular and Thoracic Surgery, Anaesthesia and Surgical Intensive Care, University Hospital Felix Guyon, Saint Denis, Reunion Island, France; ${ }^{36}$ Department of Thoracic Surgery, Cangzhou Central Hospital, Cangzhou, China; ${ }^{37}$ Department of Thoracic Surgery, Lanzhou University Second Hospital, Lanzhou University Second Clinical Medical College, Lanzhou, China; ${ }^{38} 2 \mathrm{nd}$ Department of Thoracic Surgery, the Third Affiliated Hospital of Kunming Medical University, Kunming, China; ${ }^{39}$ Department of Thoracic Surgery, Ruijin Hospital, Shanghai Jiao Tong University School of Medicine, Shanghai, China; ${ }^{40}$ Department of Thoracic Surgery, Zhengzhou Key Laboratory for Surgical Treatment for End-stage Lung Disease, Henan Provincial People's Hospital, Zhengzhou University People's Hospital, Henan University People's Hospital, Zhengzhou, China; ${ }^{41}$ Department of Thoracic Surgery, Peking University Shenzhen Hospital, Shenzhen, China; ${ }^{42}$ Department of Cardiothoracic Surgery, Zhongshan City People's Hospital, Zhongshan, China; ${ }^{43}$ Department of Thoracic Surgery, Union Hospital, Tongji Medical College, Huazhong University of Science and Technology, Wuhan, China; ${ }^{44}$ Department of Thoracic Surgery, Gaozhou People's Hospital, Gaozhou, China; ${ }^{45}$ Department of Thoracic Surgery, Cancer Hospital of China Medical University, Liaoning Cancer Hospital \& Institute, Shenyang, China; ${ }^{46}$ Department of Thoracic Surgery, Second Affiliated Hospital of the University of South China, Hengyang, China; ${ }^{47}$ Department of Cardiothoracic Surgery, Dongguan People's Hospital, Dongguan, China; ${ }^{48}$ Department of Thoracic Surgery, Shanghai Chest Hospital, Shanghai Jiao Tong University, Shanghai, China; ${ }^{49}$ Department of Thoracic Surgery, Affiliated Tumor Hospital of Guangxi Medical University, Nanning, China; ${ }^{50}$ Department of Thoracic Surgery, The University of Hong Kong-Shenzhen Hospital, Shenzhen, China; ${ }^{51}$ Department of Thoracic Surgery, the First People's Hospital of Yunnan Province, the Affiliated Hospital of Kunming University of Science and Technology, Kunming, China; ${ }^{52}$ Department of Thoracic Surgery, Policlinico Tor Vergata University of Rome, Rome, Italy; ${ }^{53}$ Department of Thoracic Surgery, Third People's Hospital of Shenzhen, Shenzhen, China; ${ }^{54}$ Department of Thoracic Surgery, Klinik am Park, Klinikum Westfalen, Lünen, Germany; ${ }^{55}$ Department of Thoracic Surgery, Taizhou Hospital, Taizhou, China; ${ }^{56}$ Department of Thoracic Surgery, Ningbo medical center LIHUILI Hospital, Ningbo, China; ${ }^{57}$ Thoracic and Cardiovascular Surgery, Ewha Womens University Seoul Hospital, Seoul, Korea; ${ }^{58}$ Department of Thoracic Surgery, The Third Affiliated Hospital of Chongqing Medical University, Chongqing, China; ${ }^{59}$ Department of Thoracic Surgery, People's Hospital of Shenzhen, Shenzhen, China; ${ }^{60}$ Department of Thoracic Surgery, Zhejiang Provincial People's Hospital, People's Hospital of Hangzhou Medical College, Hangzhou, China; ${ }^{61}$ Department of Thoracic Surgery, Hebei General Hospital, Shijiazhuang, China; ${ }^{62}$ Department of Cardiothoracic, The First Affiliated Hospital of Guangzhou University of Chinese Medicine, Guangzhou, China; ${ }^{63}$ Department of Cardio-Thoracic Surgery, Hunan Provincial People's Hospital and The First Affiliated Hospital of Hunan Normal University, Changsha, China; ${ }^{64}$ Department of Thoracic Surgery, The People's Hospital of Liaoning Province, Shenyang, China; ${ }^{65}$ Department of Thoracic Surgery, Huizhou Municipal Central Hospital, Huizhou, China; ${ }^{66}$ Department of Thoracic Surgery, Jiangxi Provincial People's Hospital Affiliated to Nanchang University, Nanchang, China; ${ }^{67}$ Department of Thoracic Surgery, The Affiliated Cancer Hospital of Xiangya School of Medicine, Central South University, Changsha, China; ${ }^{68}$ General Hospital of Southern Theater Command, PLA, Guangzhou, China; ${ }^{69}$ Department of Thoracic Surgery, Harbin Medical University Cancer Hospital, Harbin, China; ${ }^{70}$ Department of Thoracic Surgery, Anhui Chest Hospital, Hefei, China; ${ }^{71}$ Department of Thoracic Surgery, Quanzhou First Hospital Affiliated to Fujian Medical University, Quanzhou, China; ${ }^{72}$ Department of Thoracic Surgery, First Affiliated Hospital of China Medical University, Shenyang, China; ${ }^{73}$ Department of Thoracic Surgery, The Second Peoples' Hospital of Yibin, Yibin, China; ${ }^{74}$ Department of Thoracic Surgery, The First People's Hospital of Foshan, Foshan, China; ${ }^{75}$ Department of Thoracic and Cardiovascular Surgery, The First People's Hospital of Jingmen, Jingmen, China; ${ }^{76}$ Department of Thoracic Surgery, Affiliated Hospital of Xuzhou Medical University, Xuzhou, China; ${ }^{77}$ Department of Thoracic Surgery, the Affiliated Hospital of Inner Mongolia Medical University, Hohhot, China; ${ }^{78}$ Department of Thoracic Surgery, The Affiliated Luoyang Central Hospital of Zhengzhou University, Luoyang, China; ${ }^{79}$ Department of Thoracic Surgery, Qingdao Municipal Hospital, Qingdao, China; ${ }^{80}$ Department of Thoracic Surgery, Jieyang People's Hospital, Jieyang, China; ${ }^{81}$ Department of Chest Surgery, Affiliated Cancer Hospital \& Institute of Guangzhou Medical University, Guangzhou, China; ${ }^{82}$ Department of Thoracic Surgery, the Affiliated Hospital of Medical School, Ningbo University, Ningbo, China; ${ }^{83}$ Department of Thoracic Surgery, The First Affiliated Hospital of Wannan Medical College (Yijishan Hospital), Wuhu, China; ${ }^{84}$ Department of Thoracic Surgery, Zhejiang Hospital Zhejiang University School of Medicine, Hangzhou, China; ${ }^{85}$ Department of Thoracic Surgery, The Fourth Affiliated Hospital of Harbin Medical University L, Harbin, China; ${ }^{86}$ Department of Thoracic Surgery, Key Laboratory of Carcinogenesis and Translational Research (Ministry of Education), Peking University Cancer Hospital \& Institute, Beijing, China

Correspondence to: Jianxing He. Department of Thoracic Surgery and Oncology, The First Affiliated Hospital of Guangzhou Medical University, Guangzhou 510120, China; State Key Laboratory of Respiratory Disease, Guangzhou 510120, China; National Clinical Research Center for Respiratory Disease, Guangzhou 510120, China; Guangzhou Institute of Respiratory Health, Guangzhou 510120, China. Email: drjianxing.he@gmail.com. 
Submitted Jun 18, 2021. Accepted for publication Aug 23, 2021.

doi: $10.21037 /$ tlcr-21-663

View this article at: https://dx.doi.org/10.21037/tlcr-21-663

\section{Background}

The introduction of low-dose computed tomography (LDCT) for lung cancer screening has resulted in an increase of ground-glass nodules (GGNs) detection. These GGNs present as hazy increase in attenuation on lung CT, with preservation of intact bronchial and vascular structures (1). They may be malignant (preinvasive and invasive adenocarcinoma) or benign (inflammation, focal interstitial fibrosis, or hemorrhage) (2). Follow-up imaging is usually recommended for these lesions. Those GGNs that persistent or grow in size during interval scans are more likely to be malignant $(3,4)$. Therefore, histological confirmation via radiological biopsy or surgical resection is usually recommended. Depending on the extent of nodular/ solid component, it may not always be possible to perform radiological biopsy, and surgical resection for diagnosis may be required for these lesions.

Studies have also revealed that malignant GGNs are associated with excellent long-term survival, especially for pure GGNs (4-7). A recent meta-analysis has shown that malignant GGNs represent an indolent subtype of lung cancer that seldom metastasizes to mediastinal lymph nodes or other organs $(8,9)$. According to the eighth Edition of the TNM Classification for Lung Cancer published by The International Association for the Study of Lung Cancer (IASLC), the 5-year survival rate of patients with lung cancer depends on the specific stage of the disease and varies between $92 \%$ for stage IA to $10 \%$ and $0 \%$ for the stages IVA and IVB, respectively (10). Similarly, Hattori et al. (11) demonstrated that the 5-year overall survival of stage IA lung adenocarcinoma was $91.2 \%$ when the lesions presented as GGNs. Therefore, early detection, diagnosis, and treatment of lung cancer are crucial for improving lung cancer patients' survival rates.

For GGNs and early-stage lung cancer, resection by video-assisted thoracic surgery (VATS) is becoming the preferred surgical approach, owing to its minimal invasiveness. With the development of advanced VATS and novel anesthesia protocols, it is feasible now to perform spontaneous ventilation VATS (SV-VATS) without intubation in the management of wedge lung resection, mediastinal mass resection, anatomical lung segmentectomy, lobectomy, mediastinal mass resection and even more complex surgeries including tracheal resection and sleeve lobectomy (12-19). Compared with traditional intubated VATS, SV-VATS can significantly mitigate the adverse effects of mechanical ventilation, avoid potential injury associated with intubation, and avoid the side-effects of muscle relaxants. This aids the faster recovery of respiratory muscle function and lower the incidence of postoperative morbidity $(16,20)$. Furthermore, the rise in D-dimer after SV-VATS is less compared with traditional approach, suggesting that SV-VATS may have potential beneficial effects in reducing the risk of thromboembolism (13).

Recently, we have put forward the concept of tubeless VATS to describe SV-VATS procedure in which intraoperative intubation as well as post-operative chest tube placement and urinary catheterization are avoided. The aims are to simplify the surgical steps, hopefully reduce patient's post-operative discomfort and allow them to recover quicker. We have previously reported the successfully application of this approach for pulmonary nodules and several other carefully selected conditions safely, and demonstrated its potential in reducing postoperative pain and facilitating patient recovery $(20,21)$. However, relevant literature on this approach for GGNs remains limited. Therefore, this consensus statement aims to summarize the relevant technical points to facilitate a safer and broader application of this novel surgical strategy in the context of GGNs.

\section{Indications}

\section{Inclusion criteria}

Indications for tubeless VATS in GGN patients should be discussed in a multidisciplinary tumor board based on the relevant international or local guidelines (22-26).

Eligibility criteria for performing tubeless VATS include the following:

(I) Wedge resection or sub-lobar resection is considered as the primary surgical strategy. The operation ideally should not exceed 2 hours;

(II) No absolute age limit;

(III) Patients with Eastern Cooperative Oncology Group 
(ECOG) physical performance score of $\leq 2$ points;

(IV) Patients with an American Society of Anesthesiologists Standard (ASA) grade of $\leq$ III;

(V) Body mass index (BMI) $<30 \mathrm{~kg} / \mathrm{m}^{2}$;

(VI) Patients with no clinically significant cardiac history, such as ischaemic heart disease, valvular heart disease, rhythm disturbances such as frequent atrial fibrillation or premature ventricular contractions (PVCs). Patients with significant cardiac history should be optimized according the relevant guidelines before surgery is considered;

(VII) Normal cardiopulmonary function [predicted forced expiratory volume in the first second $(\mathrm{FEV} 1 \%)>50 \%$ and ejection fraction $(\mathrm{EF})>50 \%$ of predicted value]. Resting blood gas analysis showing arterial partial pressure of oxygen $\left(\mathrm{PaO}_{2}\right)$ $\geq 75 \mathrm{mmHg}$ and arterial partial pressure of carbon dioxide $\left(\mathrm{PaCO}_{2}\right)<45 \mathrm{mmHg}$;

(VIII) Normal renal function and no history of urological problems.

\section{Exclusion criteria}

(I) Patient-related factors:

(i) Patients who refuse surgery and/or the anesthesia protocols;

(ii) Coagulopathy, hypoxemia $\left(\mathrm{PaO}_{2}<60 \mathrm{mmHg}\right)$, hypercapnia [arterial carbon dioxide tension $\left(\mathrm{PaCO}_{2}\right)>50 \mathrm{mmHg}$, or elevated risk of regurgitation ( $<6$ hours of fasting) preoperatively or combined with gastroesophageal reflux disease; neurological disorders;

(iii) Severe acute pulmonary infection or tuberculosis;

(iv) Relative contraindications: upper airway secretions or persistent cough, spinal deformity, or brain edema (if thoracic epidural anesthesia is to be used);

(v) History of ipsilateral surgery and other conditions which can result in extensive pleural adhesion;

(vi) Phrenic nerve paralysis on the non-operated side should be viewed as a contraindication to VATS;

(vii) History of urological conditions increasing the need for urinary catheterization;

(viii) Significant cardiac history.

(II) Anesthesia-related factors:

(i) Allergy to local anesthesia;

(ii) Any contraindication to the regional anesthesia technique;

(iii) Difficult airway management.

(III) Surgery-related factors:

(i) Extensive pleural adhesions;

(ii) Inexperienced surgical and/or anesthesia team;

(iii) Previous ipsilateral lung surgery;

(iv) Pre-operative pleural effusion; emphysematous lung, residual bulla.

\section{Preoperative management}

\section{Preoperative evaluation}

(I) Clinical assessment: medical history of present and previous medical conditions, current symptoms, personal and family history;

(II) Laboratory tests: testing for blood type, routine blood tests, coagulation function, liver function, kidney function, infectious disease detection (hepatitis B virus, hepatitis $\mathrm{C}$ virus), electrolytes, arterial blood gas analysis, and routine urine tests;

(III) Pulmonary function: all patients should test pulmonary function;

(IV) Electrocardiogram (ECG) and in selected instances, patients with suspected cardiac conditions, cardiac echocardiogram should be performed pre-operatively;

(V) High-resolution CT (HRCT): for small or deeply located lesions, pre-operative localization is recommended, using either CT-guidance (hookwire, microcoil, methylene blue, medical adhesive, etc.), electromagnetic navigation bronchoscopy or, intraoperative ultrasound. Three-dimensional reconstruction for pulmonary vessels and bronchus can also be helpful;

(VI) For GGN\% < $75 \%$ or greater than $1 \mathrm{~cm}$, Brain nuclear magnetic resonance imaging (MRI), additional upper abdomen CT, whole-body bone scintigraphy/ whole body perfusion CT, and positron emission tomography (PET) scan examination is recommended to exclude distant metastases.

\section{Weight loss}

For patients with a preoperative $\mathrm{BMI} \geq 30 \mathrm{~kg} / \mathrm{m}^{2}$, endocrine therapy and diet may be used to achieve weight loss, optimize protein level and reduce body fat, especially abdominal fat. This can improve diaphragm function, and promote the postoperative recovery of pulmonary function $(27,28)$. 


\section{Anesthesia}

Tubeless VATS procedures are very demanding and challenging. Therefore, tubeless VATS needs to be performed by anesthesiologists well-trained and experienced with these SV-VATS anesthesia protocols (29).

\section{Anesthesia metbods}

Intravenous anesthesia combined with local intercostal nerve block is the most used method. Alternative anesthesia methods include intravenous anesthesia combined with thoracic epidural analgesia, or the thoracic paravertebral block (TPVB). The following protocol is our institutional standard: laryngeal mask airway (LMA) + incision block + intravenous anesthesia + T2-T11 intercostal nerve block + visceral pleural surface anesthesia + thoracic vagus nerve block on the surgical side (nasal cannula ventilation or facial mask ventilation can be employed as an alternative to LMA in some patients).

A useful adjunct to tubeless thoracic surgery is opioid free anesthesia (OFA) with the use of an analgesic dose of ketamine $0.05-0.1 \mathrm{mg} / \mathrm{kg} / \mathrm{h}$, dexamethasone analgesic dose, magnesium sulphate infusion, clonidine as analgesia in case of hypertension and continuous intravenous xylocaine for analgesia below the total toxic range of all local anesthetics applied. OFA can add to analgesia in case that regional anesthesia does not completely cover the operating site. Furthermore, there are also options of a spinal one-shot opioid analgesia done prior surgery in addition to OFA apart from the usual regional blocks utilised in tubeless thoracic surgery/non-intubated thoracic surgery. In addition, hypnosis can be used as a pre, perioperative and postoperative aid for tubeless thoracic surgery.

\section{Intercostal nerve block}

Intercostal nerve block: the T2-T11 intercostal nerves are blocked with $1-\mathrm{mL}$ mixture of $0.75 \%$ ropivacaine and $2 \%$ lidocaine (1:1) under VATS.

\section{Visceral pleural surface anesthesia}

After entering the thoracic cavity, $5-10 \mathrm{~mL}$ of $2 \%$ lidocaine is sprayed onto the visceral pleural surface.

\section{Vagus nerve block on the surgical side}

The vagus nerve is blocked with a $2.5-\mathrm{mL}$ mixture of $2 \%$ lidocaine and $0.75 \%$ ropivacaine to minimize the cough reflex and stabilize the surgical field.

Vagus nerve block operation site:

(I) Right thoracic cavity: on the surface of the trachea above the azygos arch;

(II) Left thoracic cavity: on the surface of the ascending aorta above the hilum (under the mediastinal pleura).

\section{Anesthesia monitoring setup}

ECG, heart rate (HR), respiratory rate (RR), pulse oxygen saturation $\left(\mathrm{SpO}_{2}\right)$, noninvasive blood pressure (BP), and Bispectral Index (BIS) are continuously monitored after the patient enters the operating room. Invasive blood pressure (IBP) and blood gases analysis are monitored via the radial artery from the arm of the contralateral side of lung surgery.

\section{Anesthesia stages}

(I) Induction of anesthesia: target-controlled infusion (TCI) of propofol $2-4.0 \mu \mathrm{g} / \mathrm{mL}$ and sufentanil $0.1-$ $0.2 \mu \mathrm{g} / \mathrm{kg}$ is used to induce anesthesia. When the BIS value falls to 60 or lower, LMA can be placed, with no muscle relaxant is given;

(II) Maintenance of anesthesia: TCI of propofol 1.5$3.5 \mu \mathrm{g} / \mathrm{mL}$ and remifentanil $0.03-0.1 \mu \mathrm{g} / \mathrm{kg} / \mathrm{min}$ is adopted to maintain anesthesia. Dexmedetomidine $0.3-1 \mu \mathrm{g} / \mathrm{kg} / \mathrm{h}$ is also used to reduce respiratory suppression while maintaining adequate anesthesia. Dexmedetomidine is stopped when the pleural cavity is closed, and propofol and remifentanil are withdrawn immediately when the skin closure begins;

(III) Ventilation: the LMA is connected to the anesthesia machine allowing assisted-controlling and monitoring of the airway. Oxygen is given at a rate of $2-3 \mathrm{~L} / \mathrm{min}$ via LMA, and fraction of inspired oxygen $\left(\mathrm{FiO}_{2}\right)$ is adjusted between $50 \%$ and $100 \%$. During the anesthesia period, if $\mathrm{SpO}_{2}$ drops to below $90 \%$ or $\mathrm{PaCO}_{2}$ increases to $60 \mathrm{mmHg}$ or higher, infusion rates of anesthetics should be adjusted first, followed by synchronized intermittent mechanical ventilation (SIMV), other modes of assisted ventilation, or manual ventilation for improving oxygenation. If hypoxemia or hypercapnia persists despite the above maneuvers, a conversion to intubation is usually required. Other studies also supported the use of face mask or nasal prong or nasal high flow oxygen therapy during SVVATS (30); 
(IV) Intraoperative monitoring includes continuous monitoring of ECG, HR, RR, $\mathrm{SpO}_{2}, \mathrm{BP}$ and the pressure of end-tidal carbon dioxide $\left(\mathrm{P}_{\mathrm{ET}} \mathrm{CO}_{2}\right)$.

\section{Timing of LMA withdrawal}

(I) The patient is awake and able to open his/her eyes when aroused (Ramsay score $\leq 2$ );

(II) Inhalation of air through the LMA: $\mathrm{SpO}_{2}>95 \%$ for 5-10 min (or $\mathrm{PaO}_{2}>85 \mathrm{mmHg}, \mathrm{PaCO}_{2}<50 \mathrm{mmHg}$ );

(III) Tidal volume (VT) $>6-8 \mathrm{~mL} / \mathrm{kg}$;

(IV) Hemodynamic parameters are stable.

\section{Conversion to other anesthesia modes}

(I) Conversion conditions:

(i) Hypoxemia: $\mathrm{SpO}_{2}<85 \%$ or hypercapnia $\mathrm{PaCO}_{2}$ $\geq 80 \mathrm{mmHg}$, which does not improve despite trying SIMV, and is associated with any of the following criteria:

(a) Haemodynamic instability: HR $>100 \mathrm{bpm}$ or a systolic pressure change of $>30 \%$ compared with the baseline value, leading metabolic causes on blood gases analysis (see below) or need for inotropic support;

(b) Arrhythmia not caused by surgical stimulation (e.g., frequent atrial or ventricular premature beats $\geq 6$ times $/ \mathrm{min}$ );

(c) $\mathrm{pH}$ value $<7.15$ at two arterial blood gas analyses (performed at intervals of 15 minutes or more).

(ii) Excessive bulging of the surgical field making operation difficult that does not improve after medical treatment, and a duration of $>5$ minutes;

(iii) Significant increase in tracheal secretions and bloody secretions, leading to difficulty breathing and increased airway obstruction. An irreversible VT decrease of $>30 \%$ during spontaneous breathing, with a peak pressure of $>20 \mathrm{cmH}_{2} \mathrm{O}$ during mechanical ventilation;

(iv) Persistent cough ( $>2$ times/min) despite visceral pleural surface anesthesia and vagus nerve block.

(II) Conversion strategy to intubated approach:

Preoperatively, before positioning the patient on the operating table, an additional bed sheet must be positioned under the back of the patient to allow rapid change of positioning from the lateral to the dorsal position. This will allow better intubation conditions during emergency.
Before turning the patient to the back, the surgeon can close a small VATS incision within a few seconds with one thick silk stitch.

Before the procedure, the scrub nurse needs to a few large sterile surgical drapes available on stand-by. These drapes should preferably be transparent and can be used to cover the incised thorax rapidly in order to keep the wound sterile.

(i) Single-lumen endotracheal tube + bronchial occlusion device (preferred);

(ii) Double-lumen bronchial catheter (if lung isolation is required due to bleeding in the airway, doublelumen endotracheal intubation is recommended).

(III) Tracheal intubation of patients in the lateral position:

(i) The head and neck placed parallel to the central axis of the body, or shake the table to the supine position 30 degrees;

(ii) The head is supported with a small square pillow to make the anterior and inferior sides of the mouth and nose more accessible;

(iii) Video laryngoscopy is recommended;

(iv) Application of catheter shaping;

(v) Close cooperation of two staff members trained in SV-VATS is required.

\section{Surgical procedure}

\section{Antibacterial prophylaxis}

Prophylactic antibiotics should be administered is intravenously administered 30 minutes to 1 hour prior to skin incision. The choice and duration of antibiotics is determined by local departmental policy.

\section{Surgical steps}

The patient is placed in a lateral position, and the upper arm is abducted and fixed on an arm supporting frame. Incision block is given after general anesthesia. Uniportal VATS is the preferred for the tubeless wedge resection. After the thoracic cavity is entered, visceral pleural surface anesthesia, intercostal nerve block, and vagus nerve block are performed.

The lesion is resected via wedge resection guided by preoperatively localization if required. The adequacy of the biopsy specimen and surgical resection margins should be confirmed by intraoperative frozen section. If necessary (e.g., the lesion is not in the resected specimen, frozen section 
indicates invasive carcinoma, etc.), the surgeon may proceed to an anatomical resection (lobectomy or segmentectomy) and lymph node dissection according to the intraoperative frozen section results. In such cases, postoperative chest drain tube drainage is usually recommended. At the end of the lung resection, the surgeon can assess whether chest tube is still required, depending on the amount of air-leak and bleeding. Ideally, all bleeding points should be stopped and air leak minimized before chest closure.

Once hemostasis is achieved and there is no major air leak, the parietal pleura is first closed leaving a gap for inserting a temporary chest tube to facilitate the expulsion of any residual air or fluid. This is achieved by connecting the chest tube an underwater seal and applying low pressure negative suction temporarily. Concurrently, the anesthesiologist will facilitate the lung re-expansion by performing lung recruitment maneuvers. Once there is no further bubble in the underwater seal and the water column fluctuates between 8 and $10 \mathrm{~cm}$ above the water surface, the temporary chest tube can be removed as this indicates that there is no air leak from the lung parenchyma. The "pulsestring" suture around the drain is tightened to close the drain incision. Then, the muscle and the skin are sutured.

\section{Surgical precautions}

(I) During the operation (especially before the vagus nerve block), traction of lung tissue should be avoided, especially around the hilar structures. More gentle maneuvers, such as pushing and pulling, should be applied, while rougher actions, such as pressing and lifting, should be avoided. Excessive traction around the hilum may cause a coughing reflex and increase the risk of mediastinal or diaphragmatic bulging.

(II) Sterile water should be used for testing air leaks. The warm fluid should not be poured rapidly into the thoracic cavity, as this may stimulate the pleura or vagal nerve, which might increase the risk of diaphragmatic bulging or even induce cardiac arrest.

(III) In some cases, the lung lobe where the lesion(s) is/ are removed, may not fully reinflate during lung recruitment procedures. This may be due to the decreased compliance of the lobe after the resection or the leakage around the LMA. Negative pressure can be used via the drain tube to assist lung recruitment maneuvers by the anesthesiologists. Airway secretion should also be excluded. If the lung still does not fully re-inflate, a chest tube should be considered.
(IV) The operator should assess whether an indwelling chest tube can be avoided, according to surgical situations and disease conditions. If there is no air leak, or post-operative oozing, and the remaining lung is fully re-expanded, indwelling chest tube can be avoided.

(V) If the mediastinal swing's too excessive especially during dissection of vital structures around the hilum, a small dose (how many dose?) of non-depolarization muscle relaxants and SIMV mode with low VT can be given.

\section{Intraoperative complication management}

\section{Intraoperative cough}

A cough reflex is mainly induced by compressing the bronchial cartilage or stretching the hilum, which may negatively affect the surgical procedure. Vagus nerve block and visceral pleural surface anesthesia at the surgical side can effectively inhibit the cough reflex.

\section{Mediastinal swing}

Excessive mediastinal swing may negatively affect operational maneuvering because of one-lung spontaneous ventilation and the effects of anesthetic drugs on breathing after establishing iatrogenic pneumothorax. Ideal parameters for one-lung spontaneous ventilation are RR $10-15$ beats $/ \mathrm{min}$ and VT $3-4 \mathrm{~mL} / \mathrm{kg}$. In this range, the breathing rhythm of patient is regular, mainly chest breathing, and the mediastinum swings slightly, which does not affect the operation. However, excessive swinging of the mediastinum will affect the operation and damage vessels.

Causes of excessive mediastinal swing and corresponding treatments: (I) inadequate analgesia, such as paravertebral nerve block, imperfect intercostal block, and patients often have irregular breathing. The anesthetist can increase the dose of remifentanil and the surgeon may add intercostal nerve block; (II) anesthesia is too deep, respiratory depression, mostly due to the effects of propofol and remifentanil, the drug speed can be adjusted appropriately; (III) $\mathrm{CO}_{2}$ accumulation, patients show deep breathing, which can be assisted with SIMV mode; (IV) with incomplete vagus nerve block the patient exhibits choking and mediastinal swing during the operation. Further vagus nerve block can be administered.

\section{Intraoperative hypoxemia}

After the operated lung collapses, the surgical side's airway 
resistance would be higher than that of the contralateral side. Therefore, most ventilated gases enter the contralateral lung during the low VT/pressure ventilation. The non-dependent (operated) lung is thus seldom inflated and negligibly impacts the surgical procedures. At the same time, blood shunt is improved by gravity and volume reduction of the operated lung, resulting in better oxygenation in the contralateral lung and a higher $\mathrm{SpO}_{2}$. Although the incidence of hypoxemia is low, anesthesiologists should be familiar with the treatment in this case. If $\mathrm{SpO}_{2}$ drops to $90 \%$ or lower, SIMV (VT 3-5 mL/kg, RR 12-15 times/min), up-regulated $\mathrm{FiO}_{2}$ and oxygen flow or manually assisted ventilation can be applied.

\section{Intraoperative hypercapnia}

If $\mathrm{PaCO}_{2}$ rises to $60 \mathrm{mmHg}$ or more, infusion rate of propofol can be adjusted in conjunction with SIMV (VT 3-5 mL/kg, RR 12-15 times $/ \mathrm{min}, \mathrm{FiO}_{2}=100 \%$, oxygen flow $4-5 \mathrm{~L} / \mathrm{min}$ ) or manual ventilation. If these treatments fail to reverse hypercapnia, and the $\mathrm{PaCO}_{2}$ rises above $80 \mathrm{mmHg}$ conversion to intubated surgery should be considered. The timing of anesthesia conversion is not based on duration of surgery, but depends on the patient's tolerance status, as detailed in the conversion conditions mentioned above.

\section{Intraoperative airway management}

(I) The migration of the LMA: if the patient suddenly experiences inspiratory dyspnea during operation, VT suddenly drops, or the $\mathrm{P}_{\mathrm{ET}} \mathrm{CO}_{2}$ waveform suddenly becomes flat or disappears, the position of LMA should be checked. In this situation, the LMA needs to be appropriately adjusted to the correct position under deeper anesthesia;

(II) Airway leakage after lung re-inflation: if there is air leakage from the LMA, reinflation of the operated lung may be difficult. Leakage can be reduced by gently pressing both sides of the thyroid cartilage or by repositioning the LMA, and occasionally by bronchoscopy to ensure correct placement;

(III) Management of intraoperative sputum suction: During the surgery, if sputum suction is required via LMA, the negative pressure should be $<10 \mathrm{kPa}$, and each sputum aspiration time should be $<10 \mathrm{~s}$. Repeat stimulation of the glottis should be avoided as it may cause laryngeal spasm and coughing. Application of lidocaine spray over the upper airways may be beneficial.

\section{Postoperative management}

Routine postoperative monitoring and investigations

(I) Vital signs (HR, RR, BP, $\mathrm{SpO}_{2}$ ) are measured continuously for 12 hours postoperatively. Subjective symptoms include pain, chest tightness, shortness of breath, and lung signs should be recorded;

(II) Full blood count and renal panel should be considered if there is significant blood loss during surgery;

(III) A chest X-ray (CXR) is performed within 8 hours after surgery. Thoracic ultrasound could be performed every 8 hours within 24 hours postoperatively to evaluate pleural effusion. A second CXR reexamination and chest ultrasound scan for pleural effusion are performed within 48 hours after surgery.

\section{Evaluation of lung reinflation}

(I) Evaluation method: a CXR is performed within 8 and 48 hours after surgery;

(II) Evaluation criteria: according to the degree of postoperative lung recovery, lung reinflation can be divided into poor (lung reinflation $<50 \%$ ), medium $(70 \%>$ lung reinflation $\geq 50 \%$ ), and good (lung reinflation $\geq 70 \%)$.

\section{Evaluation of the need for postoperative thoracic tube replacement}

Small amounts of pleural effusion or pneumothorax should normally reabsorb itself without the need for thoracentesis or reinsertion of the chest tube. However, if there is poor lung re-expansion on CXR or a moderate to large pleural effusion on CXR and chest ultrasound, chest tube insertion is recommended. In cases of poor lung re-expansion, atelectasis/mucus plug (see below) should be excluded concurrently. In instances of moderate pneumothorax or pleural effusion, it may be possible to try thoracentesis first before deciding if a chest tube insertion is still required.

\section{Indications and methods for the chest tube withdrawal}

(I) CXR or chest CT scan shows good lung expansion (no residual cavity in the chest cavity);

(II) No bubbles emerge from the drainage bottle during deep inspiration or coughing. The drainage fluid is not bloody, chylous, or purulent;

(III) The volume of chest drainage is $\leq 200 \mathrm{~mL} / 24$ hours.

\section{Indications for urinary catheter}

(I) Patients developed post-operative urinary retention, 
as confirmed on bladder scan;

(II) Haemodynamic instability during surgery, due to cardiorespiratory instability or intraoperative bleeding;

(III) Relative indications-prolonged operation $>2$ hours.

\section{Use of antibiotics}

The use of postoperative antibiotics is determined by local guidelines.

(I) Criteria for 2 nd dose of prophylactic antibiotics (30-32): normally, only 1 antibiotic dose is given half an hour before surgery; the second dose of the antibiotic drug should be considered in the following conditions:

(i) The operation lasts more than 3 hours;

(ii) Intraoperative massive blood loss is $>1,500 \mathrm{~mL}$.

(II) Criteria for starting therapeutic doses of antibiotics:

(i) Clinical evidence of pleural infection, e.g., purulent pleural aspiration/effusion;

(ii) Body temperature is higher than $38.5^{\circ} \mathrm{C}$;

(iii) There is purulent excretion in the airway;

(iv) Postoperative white blood cell (WBC) count is $\geq 11 \times 10^{9} / \mathrm{L}$;

(v) The procalcitonin level is higher than $0.5 \mathrm{ng} / \mathrm{mL}$. If 3 of the above criteria are met, the antibiotics can be reused until the above criteria for drug withdrawal are met again.

\section{Postoperative pain management}

Pain assessment is based on the international pain score and performed within 24 hours after surgery.

(I) The scoring criteria [Visual Analogue Scale (VAS) (0-10 points) (33-35)]:

(i) 0 points, no pain;

(ii) $\leq 3$ points, slight pain that is endurable;

(iii) 4-6 points, pain interferes with sleep but may still be endured;

(iv) 7-10 points, increasingly severe pain that cannot be endured, interferes with appetite and sleep.

(II) Analgesic application

(i) Nonsteroidal anti-inflammatory drugs (NSAIDs) can be used for mild pain (VAS 1-3 points);

(ii) Weak opioids and NSAIDs can be used for moderate pain (VAS 4-6 points);

(iii) Potent opioids and NSAIDs can be used for severe pain (VAS 7-10 points).
Guidance of postoperative feeding and ambulation

(I) A small amount of liquid diet can be started 4-6 hours after surgery, and water quantity can be gradually increased. If the patient does not experience nausea or vomiting during this period, a semifluid diet can be started, followed by a gradual transition to a regular diet.

(II) Postoperative ambulation: after fully waking up from anesthesia, patients are encouraged to ambulate with the help of the nursing staff or family members as soon as possible. Early ambulation may reduce the incidence of complications such as atelectasis and venous thromboembolism.

\section{Postoperative complications and management specific to tubeless VATS}

Common complications include the following:

(I) Atelectasis: atelectasis may be caused by blood or mucus plugging of the airway during surgery or early postoperatively. Patients are encouraged to actively cough to clear the sputum. Moreover, intensive physiotherapy is also given to patients after surgery to optimize lung reinflation. Atomization inhalation can be used to assist sputum excretion. If necessary, fiberoptic bronchoscopic secretion clearance can be considered in selected cases.

(II) Poor lung reinflation: this may be caused by air leaks or inadequate postoperative suctioning with negative pressure or atelectasis. If necessary, the drain tube should be reinserted concurrently with treatment for atelectasis. Adequate nutritional support, constant low negative-pressure suction $(<2 \mathrm{kPa})$ in the thoracic cavity or biphasic intermittent positive airway pressure (BiPAP)assisted ventilation (2 hours/bid) may support lung reinflation.

(III) Pleural effusion: as there is no chest tube available to monitor pleural effusion, patients should be monitored in regularly with pleural ultrasound to exclude postoperative pleural effusion. This should be co-related with patient's clinical condition and haemoglobin level to exclude post-operative intra-thoracic bleeding. If there are concerns with bleeding, a chest CT scan with contrast or even a second operation should be performed.

(IV) Urinary retention: theoretically, the avoidance of muscle relaxants should reduce the incidence of acute urinary retention. However, if patient develops acute postoperatively urinary retention, 
they will require urinary catheterization.

\section{Conclusions}

GGNs can be benign or malignant. Radiological biopsy for histological diagnosis may not be always feasible and surgical resection may be required for diagnosis as well as treatment. Minimally invasive VATS approach is currently preferred for these GGNs. Traditionally, double-lumen intubation is required.

The development of SV-VATS provided an alternative approach for the management of these lesions. Previous reports indicate that SV-VATS can reduce anesthetic time and potentially accelerate postoperative recovery, by encouraging early ambulation, early nutrition and thereby minimizing associated post-operative complications (14,16,36-38). To further refine SV-VATS in line with the concept of fast-track surgery and enhanced recovery programme, tubeless VATS was started. Tubeless VATS has further refined the technique of SV-VATS to include the avoidance of post-operative chest tube and urinary catheter. Its aims are to minimize the trauma and pain associated with tube interventions. enhance postoperative recovery, and hopefully make ambulatory VATS a reality $(39,40)$.

GGNs are well-suited for tubeless VATS because most of them can be removed via sublobar resection. Even if they are malignant, they behave in biologically indolent way and rarely involved the lymph nodes $(41,42)$. Therefore, they do not require extensive surgical resection unless there is an invasive tumour component. Multiple studies have shown that patients with GGNs, whether they are pure GGNs or mixed GGNs, have a 5 -year survival rate of over $90 \%$ and a 3-year recurrence-free survival (RFS) of more than $95 \%$ after sublobar resection (11,41-43). This reduces operative time and complexity as well as risk of complications, making it favorable for the adoption of tubeless VATS approach. For these reasons, tubeless VATS can be considered a simple and reliable alternative to the conventional VATS performed by double-lumen tube intubation and singlelung mechanical ventilation anesthesia for GGN surgery.

\section{Questions}

\section{Do you apply tubeless VATS for peripheral GGO resection (wedge resection)?}

Eugenio Pompeo: Yes, we do perform tubeless wedge resection in selected patients with GGO.
Bassam Redwan: For resection of peripheral GGO nonintubated VATS (niVATS) is more frequently applied than tubeless VATS, since we have observed a higher rate of postoperative pneumothorax occurrence, especially in case of emphysematous lungs.

Paolo A. Ferrari: We do not routinely apply tubeless VATS for peripheral GGN resection. However, we always consider this approach a less invasive procedure in fragile patients when a pathological diagnostic report for lung GGNs is needed.

Alberto Aiolfi: Tubeless VATS is used in our institution for the treatment of peripheral GGO resection, pleural effusion, and bullectomy for pneumothorax.

Kyoji Hirai: Yes, I apply tubeless VATS, in case that I can get informed consent from patients.

Kyung Soo Kim: Yes, SV-VATS with tubeless, uniportal approach is primarily preferred for resection of peripheral GGO lesions with several advantages. Suggested selection criteria are compatible with my tubeless VATS procedures. For this, cooperation with consultation is routinely requested to anesthesiologists in planning tubeless surgery, even for simple wedge resection. Preoperative sono-guided paravertebral block is preferred prior to tubeless VATS, but epidural block is not introduced in our institutions.

Luca Bertolaccini: No, dependently the GGO dimension and if there is a need for an external marker.

Sook-Whan Sung: Yes, tubeless VATS is an easy and very useful modality for peripheral GGO resectional surgery.

Soichiro Funaki: Yes, I am. I think there is a possibility that tubeless VATS is applied for wedge resection for early lung cancer or tiny metastasis.

Marios E. Froudarakis: I do apply tubeless thoracoscopy but not for peripheral GGO resections, as I am only interested to treat pleural disease.

Andrey Akopov: We did not perform wedge resections for GGO. We always perform segmentectomy or lobectomy. So, we did not use tubeless surgery for GGO. But we use tubeless VATS for pulmonary biopsy (wedge resection) for patients with interstitial lung diseases.

Raja M. Flores: No, I do not.

Gabor Kiss: No, because our surgeons are not experienced in this technique.

Keng-Leong Ang: Yes.

\section{How do you select candidates for receiving tubeless VATS?}

Eugenio Pompeo: We usually deserve tubeless VATS to 
patients with advanced ( $>75$ years) age or with associated comorbidity particularly chronic obstructive pulmonary disease (COPD) or interstitial lung disease.

Bassam Redwan: In our institution, tubeless VATS is routinely applied for sympathectomy in hyperhidrosis patients. Since no manipulation of the lung tissue is necessary, risk of postoperative pneumothorax is low. When tubeless VATS for GGO resection is planned, lung emphysema should be excluded in thoracic imaging studies and in lung function testing. GGO should be localized peripherally, and patients should be compliant for the intraoperative setting of tubeless VATS. Patients with effective anticoagulation are usually excluded from tubeless VATS.

Paolo A. Ferrari: Candidates' selection depends on the surgery. However, when tubeless VATS is preferable, patients are excluded from undergoing this kind of surgery if any of the following are present: age $<18$ years, hemodynamic instability, patient already intubated and ventilated, anticipated need for extensive decortication. Moreover, according to our experience, we do not consider obesity an insurmountable barrier in tubeless VATS.

Alberto Aiolfi:

* ASA grade of $\leq$ II;

* Physical performance score according to the ECOG $\leq 1$ point;

* BMI $<30 \mathrm{~kg} / \mathrm{m}^{2}$;

* Tumor diameter <5 cm;

* Aged <60 years;

* Expected operation duration less than 2 hours;

- Patients who are unlikely to develop severe complications such as major bleeding (patients taking platelet aggregation inhibitors or anticoagulation drugs), respiratory obstruction, and severe postoperative pain;

* No prior thoracic surgery of minor procedures (i.e., transthoracic pleural biopsy)

- Normal cardiopulmonary function (EF $>50 \%$ and FEV $1 \%>50 \%$ of predicted value);

* Resting blood gas analysis without oxygen inhaling shows $\mathrm{PO}_{2} \geq 80 \mathrm{mmHg}$ and $\mathrm{PCO}_{2}<45 \mathrm{mmHg}$;

Patients without these criteria are excluded.

Kyoji Hirai: Basically, I select the patients who have good physical condition. Subsequently, I apply tubeless VATS for patients who have poor physical condition. However, as for patients who have poor physical condition, sufficient informed consent is required.

Kyung Soo Kim: Tubeless VATS are fit for non-obese patients without having severe low pulmonary functions or cardiac morbidities. Previous pleural infection is contraindicated firstly, but prior VATS history will not be contraindicated, because peripheral loose adhesions can be easily dissected to continue tubeless VATS by less stimulating techniques. Moreover, application of antiadhesive materials can be applied in conducting VATS, that tubeless redo VATS might be promising in selective cases.

Luca Bertolaccini: Young, fit for surgery, peripheral lesion.

Sook-Whan Sung: Usually, we can apply tubeless VATS in every feasible thoracoscopic cases; if patient is not so obese (less than $30 \mathrm{BMI}$ score), patient who do not have severe pleural adhesion requiring decortication; patient who has very difficult condition because of invasive central cancer and/or severe perivascular fibrosis with anthracosis.

Soichiro Funaki: I think that wedge resection of tiny metastasis, early lung cancer (small pure GGN and lymph node dissection is not needed, or unknown small nodule (for main aim of biopsy) is good candidate for tubeless VATS.

Marios E. Froudarakis: I select patients for tubeless thoracoscopy according to their disease (pleural disease such as, empyema, malignancy, pneumothorax), their performance status $(\mathrm{PS}<2)$, their comorbidities and their ability to cooperate during the procedure.

Andrey Akopov: Patients with interstitial lung diseases who underwent pulmonary biopsy (wedge resection).

Raja M. Flores: Select candidates that are too sick for general anesthesia.

Gabor Kiss: No, see answer 1. In contrast to my previous working place, our surgeons are not experienced in this technique. However, I also want to add that phrenic nerve paralysis on the non-operated side should be viewed as a contraindication to any type of non-intubated thoracic surgery which also includes tubeless VATS.

Do not forget, that tubeless thoracic surgery or its equivalent "Non incubated thoracic surgery" is an excellent indication for pregnant patients and in case of contraindication to general anesthesia mainly for patients with severe chronic pulmonary disease, lung transplant candidates and those with myopathy, allergy to muscle relaxants and a history of difficult weaning from postoperative ventilation or in the intensive care unit (ICU). Also, patients with a past medical history of pneumonectomy needing minor surgery on the only lung left are good indications for any type of non-intubated thoracic surgery

Keng-Leong Ang: Candidate must be suitable for SV-VATS, and has no risk factors requiring urine 
catheterisation and post-op chest drainage.

\section{Do you regard this surgery as more challenging under spontaneous breathing (tubeless)?}

Eugenio Pompeo: Not much more challenging provided adequate training of both surgery and anesthesia teams is achieved.

Bassam Redwan: During the initial phase of establishing niVATS at our institution, performing procedures in patients under spontaneous breathing was more challenging than conventional intubated surgery due to the lung excursions, which influence the surgical exposure, especially while performing anatomical resections. However, with growing experience, surgeons are adapted to these changes and procedures are performed in a standardized and comparable fashion to intubated surgery.

Paolo A. Ferrari: There are no dramatic differences between peripheral lung wedge VATS resection in intubated and tubeless patients from a merely technical perspective. Effective teamwork is essential to prevent any possible challenges during tubeless VATS.

Alberto Aiolfi: Yes, I do. I believe that tubeless VATS is more challenging than the standard procedure. However, in the hands of experienced surgeons and after an appropriate learning phase (to be defined in future studies) it has some important advantages in selected patients.

Kyoji Hirai: Yes, this surgery under spontaneous breathing is more challenging and promising as a next minimally invasive surgery. Naturally, the close collaboration with anesthesiologist is essential.

Kyung Soo Kim: It seems challenging in starting niVATS without placing chest tube. Careful patient selection and understanding of physiologic change is important to maintain SV-VATS as dedicated team surgery. Tubeless technique requires continuous interactions with the anesthesiologist because abrupt, unexpected situations may happen anytime. Thus, team work is relatively more required, compared to conventional anesthetic surgery under intubation. Gentle manipulation of the lung, hilum and tracheobronchial tree should be reserved in whole tubeless procedures. Minor VATS surgery can be easily applied, achieving early enhanced recovery with its efficacy, as described. Extended complex surgery has also been applied by experienced centers, but safety consensus seems still debatable for broad application and educational issues.

Luca Bertolaccini: Yes, it is time dependent.

Sook-Whan Sung: I think tubeless VATS is pretty the same as intubated VATS in aspect of feasibility and completeness of operation. However, it needs experiences and patience. Usually, 10 to 20 cases of experience are enough to be a self-confident surgeon. This is the same question raised during beginning era of VATS surgery at early 90. At that time, most thoracic surgeon thought VATS was inappropriate for resectional lung surgery except simple wedge resection for the fear of incompleteness of surgery and vascular injuries. It is a little challenging surgery. Once adopted in tubeless surgery usually within 10 to 20 cases of experience, the surgeon can do same surgery with tubeless VATS as conventional VATS. Needless to say. we need a good support from enthusiastic anesthesiologist who can manage the patient very smoothly during the operation.

Soichiro Funaki: Yes, I think tubeless VATS under spontaneous breathing is more difficult and challenging as compared to usual general anesthesia.

Marios E. Froudarakis: No, because I have a great experience, and the procedure looks easy to my eyes.

Andrey Akopov: Yes.

Raja M. Flores: Same.

Gabor Kiss: Personally, as an anesthesiologist, a tubeless technique is more challenging and requires more attention but when you know the technique, it is safe.

Keng-Leong Ang: Tubeless VATS is an extension of spontaneous breathing/SV-VATS, and it requires additional considerations outlined in this paper. Surgical and anaesthesiological team performing the procedure need to be familiar with and trained in the technique. Whether the procedure is more challenging under spontaneous breathing ultimately is dependent on the patient's anatomy and physiological factors.

\section{How do you choose between continuing tubeless VATS or converting to intubated VATS if lobectomy is required during surgery?}

Eugenio Pompeo: Whenever lobectomy is required, we more commonly perform it by intubated anesthesia. The same indications listed in point 2 are applied.

Bassam Redwan: If lobectomy is necessary, niVATS and not tubeless VATS is performed at our institution.

Paolo A. Ferrari: We always convert to intubated VATS if anatomical lung resection with complete mediastinal lymph node dissection is needed. The challenge of performing lobectomy in spontaneously breathing patients lies in need to isolate and suture delicate structures like pulmonary vessels and bronchi. Operative skills are undeniably essential 
in such interventions while trying to cope with the lack of stillness of the surgical field and the diaphragmatic and/or lung movement.

Alberto Aiolfi: This is a matter of intense debate. I believe that current evidence reporting results for major surgical procedures (i.e., lobectomy) is scarce and nonconclusive. In case a lobectomy is required because intraoperative findings, we always decide to shift toward intubated VATS.

Kyoji Hirai: The patients with massive bleeding, sputum, continuous cough or severe lung adhesion to adjacent organs during surgery should be converted to intubated VATS.

The operations that take for more than three hours and requires anatomical lung resection with complete lymph node dissection also are unstable for tubeless VATS.

Kyung Soo Kim: Tubeless lobar resection with mediastinal dissection has also been practically introduced by experienced team settings. Invasive tumor characteristics or severe hilar adhesions with anthracosis might require conversion. If abrupt inflation of the lung and coughing reflex continues, withdrawals of thoracoscopic instruments have to be promptly executed to avoid intrathoracic organ or intercostal injury. Continuing tubeless VATS should be reassessed for decision, requiring intubated VATS for more technically uneasy, time-consuming and unsafe procedures.

Luca Bertolaccini: In case of pain, dyspnea, bleeding or patient anxiety, it should be discussed with the anesthesiologist the possibility of thoracotomic conversion.

Sook-Whan Sung: I usually continue tubeless VATS for lobectomy. We frequently encounter undiagnosed lung mass. In those cases, with tubeless VATS we continued the procedure from diagnostic wedge followed by lobectomy for undiagnosed lung cancer. Also nowadays, conversion rate of endotracheal intubation is around $1-2 \%$ for experienced center. Double lumen endotracheal intubation is not so difficult during tubeless VATS surgery.

Soichiro Funaki: I think that intubated VATS should be done in any cases with lobectomy.

Marios E. Froudarakis: I do not perform surgical procedures, therefore, I have no opinion about that.

Andrey Akopov: We have no situations like this.

Raja M. Flores: Lobectomy is performed with tube.

Gabor Kiss: Conversion to intubated VATS is indicated in case of oxygen saturation $\left(\mathrm{O}_{2}\right.$ Sat $)$ below $90 \%$ in spite of high oxygen flow via mask or non-invasive ventilation (NIV), hemodynamic instability, uncontrolled surgical bleeding not answering to anesthetic intervention, uncooperative and/or anxious patient and uncontrollable cough in spite of lidocaine or vagal block.

Keng-Leong Ang: This question is rather confusing, as tubeless VATS strictly speaking included additional considerations to those of SV-VATS. Whether tubeless VATS is possible can only be determined at the end of the procedure, i.e., whether patient requires chest tube or urinary catheterization. If lobectomy is required, whether to convert from SV to intubated VATS is a different matter. Criteria used for this is already outlined in the manuscript.

\section{When do you deem it necessary to convert to intubated VATS during surgery?}

Eugenio Pompeo: We usually apply the same criteria as those well pointed out in the current text.

Bassam Redwan: Conversion criteria to intubated VATS include surgical complications, such as major bleedings, severe hypoxemia $\left(\mathrm{paO}_{2}<60 \mathrm{mmHg}\right)$, severe hypercapnia $\left(\mathrm{paCO}_{2}>80 \mathrm{mmHg}\right.$ ) with acidosis $(\mathrm{pH}<7.1)$, hemodynamic instability, persistent coughing and excessive diaphragm movement, which compromise safety of the surgical procedure.

Paolo A. Ferrari: Concerning the surgical aspects, severe bleeding, diaphragm swing, relentless inflation, and deletion of the lung are the leading cause of conversion to intubated surgery. In any case, continuous and effective communication between surgical and anaesthetic teams is paramount.

Alberto Aiolfi: In certain operations, conversion to general anesthesia and standard VATS is inevitable to preserve patient and operating surgeons' safety. Major bleeding, adhesions, significant mediastinal shift/movement, and/or persistent hypoxaemia and tachypnea, have been identified as factors associated with conversion to intubated VATS. In our center we use these criteria for conversion.

Kyoji Hirai: I take into consideration for converting to intubated VATS during operation next.

(I) Massive bleeding;

(II) Massive sputum;

(III) Continuous cough;

(IV) Hypercapnia, hypoxia, acidosis on arterial blood gas;

(V) Hemodynamic instability such as unstable BP, tachycardia, arrhythmia and ST abnormality on ECG.

Kyung Soo Kim: Most wedge resection procedures are finished within a brief time, but the additional block or the infusion of anesthetic agents may be needed to continue 
planned VATS. Minor bleedings from adherent nodes, small vessels or lung injury can be managed without conversion. Respiratory distress with arrhythmia despite of modifying ventilation setting is firstly considered. Smooth conversion should be properly carried out during hemostatic compression, even requiring thoracotomy in cases with major vessel bleeding.

Luca Bertolaccini: In case of unstable hemodynamics, dyspnea and any bleeding.

Sook-Whan Sung: Indications for conversion to intubated VATS:

(I) If the surgeon is not satisfied with tubeless VATS lobectomy;

(II) Unstable hemodynamics;

(III) Too much deep respiration causing wide excursion of mediastinum;

(IV) Severe bleeding;

(V) Severe pleural adhesion;

(VI) Anthracotic peribronchial infiltrative lymph nodes;

(VII) Complicated surgery such as bronchoplasty, sleeve resection, chest wall resection, etc.

Soichiro Funaki: I think that intubated VATS should be done when the adhesion between chest wall and lung, more advance staging could be found.

Marios E. Froudarakis: I do not perform surgical procedures, therefore, I have no opinion about that.

Andrey Akopov: Hemodynamic disorders mainly.

Raja M. Flores: For lobectomy.

Gabor Kiss: As in the previous questions, criteria are desaturation remaining below $90 \%$ on the pulse oximeter in spite of high oxygen flow or NIV, hemodynamic instability, uncontrolled surgical bleeding, uncooperative patient, uncontrollable cough but also at any time with a stable patient but on the surgeon's request in order to improve his operating conditions.

Keng-Leong Ang: It depends on patient's factorswhether the patient's anatomy is favourable for SV-VATS and whether patient can tolerate SV physiologically. We used similar criteria for conversion described in this paper.

\section{What do you think is the biggest challenge when performing tubeless VATS?}

Eugenio Pompeo: Management of incidental major bleeding if anatomical resection with hilum vascular dissection is required.

Bassam Redwan: The biggest challenge when performing tubeless VATS is the interaction between surgeons and anesthesiologists during surgery, especially in case of complications requiring conversion to intubated VATS. Intubation in lateral position should be performed by an experienced anesthesiologist. A further challenging issue is the postoperative period. Special care should be taken, to enable early detection of possible complications such as pneumothorax or bleeding. Therefore, it is obligatory to have an institutional standard protocol for postoperative monitoring.

Paolo A. Ferrari: According to our experience in performing tubeless wedge resections for GGNs, the inability to collapse the lung (paradoxically maintained by ventilation or dynamic hyperinflation) and the excessive movements of diaphragm or mediastinum are the biggest causes of unsafe surgery.

Alberto Aiolfi: I believe that the biggest challenge for tubeless VATS is to explore its safety, effectiveness, and long-term results compared to the standard VATS in the setting of major lung resections (i.e., lobectomy). Despite, a systematic review has been published recently with comparable promising results, only three retrospective studies were included with a limited number of patients $(n=204)$. Therefore, conclusions are significantly biased by studies design, patients 'selection, and limited oncologic long-term follow-up implications. A such, I believe that this is the biggest future challenge for tubeless VATS (19).

Kyoji Hirai: The biggest challenge when performing tubeless VATS is patient's safety, and to complete treatment for malignancy.

Kyung Soo Kim: Cooperative team interaction seems most challenging to initiate tubeless VATS. Oral, tooth and tracheobronchial injuries might occur during conversion procedures that informed consent should be explained prior to surgery. To avoid uneventful situations, simulation program under suggested consensus is thought to be essential, even for simple wedge by proper selection criteria.

Luca Bertolaccini: The patients' safety, since conclusions of published studies are significantly biased by studies design, patients 'selection, and limited oncologic long-term follow-up implications (19).

Sook-Whan Sung: The biggest challenge of the tubeless VATS is as follows: (I) there are not enough data of safety and feasibility and effectiveness of the operation comparing to conventional VATS. Collaborative researches are necessary. (II) Because of its beginning period, there are not enough education program on this innovative procedure. Every surgeon who is eager to do this surgery should visit renowned center to understand this procedure. 
Soichiro Funaki: I think that biggest challenge is to secure the safety and curability for malignancy.

Marios E. Froudarakis: I think that the biggest challenge is patients' comfort especially regarding pain, because this affects the physicians' comfort for a well driven procedure.

Andrey Akopov: The necessity of intubation-it is not always easy to intubate the trachea (converting to intubated VATS) in emergency situations, especially in the position of the patient on the side.

Raja M. Flores: If bleeding occurs getting exposure.

Gabor Kiss: Not manageable deoxygenation during surgery needing rapid airway management (LMA, intubation, etc.).

Keng-Leong Ang: Selection of suitable patients.

\section{Acknowledgments}

The authors appreciate the academic support from the AME Thoracic Surgery Collaborative Group.

Funding: None.

\section{Footnote}

Conflicts of Interest: All authors have completed the ICMJE uniform disclosure form (available at https://dx.doi. org/10.21037/tlcr-21-663). Wenhua L serves as an unpaid Associate Editor-in-Chief of Translational Lung Cancer Research. The other authors have no conflicts of interest to declare.

Ethical Statement: The authors are accountable for all aspects of the work in ensuring that questions related to the accuracy or integrity of any part of the work are appropriately investigated and resolved.

Open Access Statement: This is an Open Access article distributed in accordance with the Creative Commons Attribution-NonCommercial-NoDerivs 4.0 International License (CC BY-NC-ND 4.0), which permits the noncommercial replication and distribution of the article with the strict proviso that no changes or edits are made and the original work is properly cited (including links to both the formal publication through the relevant DOI and the license). See: https://creativecommons.org/licenses/by-nc-nd/4.0/.

\section{References}

1. Suzuki K, Kusumoto M, Watanabe S, et al. Radiologic classification of small adenocarcinoma of the lung: radiologic-pathologic correlation and its prognostic impact. Ann Thorac Surg 2006;81:413-9.

2. Gao JW, Rizzo S, Ma LH, et al. Pulmonary ground-glass opacity: computed tomography features, histopathology and molecular pathology. Transl Lung Cancer Res 2017;6:68-75.

3. Kim H, Goo JM, Kim YT, et al. Consolidation-to-tumor ratio and tumor disappearance ratio are not independent prognostic factors for the patients with resected lung adenocarcinomas. Lung Cancer 2019;137:123-8.

4. Hattori A, Matsunaga T, Takamochi K, et al. Importance of Ground Glass Opacity Component in Clinical Stage IA Radiologic Invasive Lung Cancer. Ann Thorac Surg 2017;104:313-20.

5. Ye T, Deng L, Wang S, et al. Lung Adenocarcinomas Manifesting as Radiological Part-Solid Nodules Define a Special Clinical Subtype. J Thorac Oncol 2019;14:617-27.

6. Hattori A, Matsunaga T, Hayashi T, et al. Prognostic Impact of the Findings on Thin-Section Computed Tomography in Patients with Subcentimeter Non-Small Cell Lung Cancer. J Thorac Oncol 2017;12:954-62.

7. Aokage K, Miyoshi T, Ishii G, et al. Influence of Ground Glass Opacity and the Corresponding Pathological Findings on Survival in Patients with Clinical Stage I NonSmall Cell Lung Cancer. J Thorac Oncol 2018;13:533-42.

8. Zha J, Xie D, Xie H, et al. Recognition of "aggressive" behavior in "indolent" ground glass opacity and mixed density lesions. J Thorac Dis 2016;8:1460-8.

9. Zhang Y, Fu F, Wen Z, et al. Segment Location and Ground Glass Opacity Ratio Reliably Predict NodeNegative Status in Lung Cancer. Ann Thorac Surg 2020;109:1061-8.

10. Goldstraw P, Chansky K, Crowley J, et al. The IASLC Lung Cancer Staging Project: Proposals for Revision of the TNM Stage Groupings in the Forthcoming (Eighth) Edition of the TNM Classification for Lung Cancer. J Thorac Oncol 2016;11:39-51.

11. Hattori A, Hirayama S, Matsunaga T, et al. Distinct Clinicopathologic Characteristics and Prognosis Based on the Presence of Ground Glass Opacity Component in Clinical Stage IA Lung Adenocarcinoma. J Thorac Oncol 2019;14:265-75.

12. Peng G, Cui F, Ang KL, et al. Non-intubated combined with video-assisted thoracoscopic in carinal reconstruction. J Thorac Dis 2016;8:586-93.

13. Liang $\mathrm{H}, \mathrm{Liu} \mathrm{J}$, Wu S, et al. Nonintubated Spontaneous Ventilation Offers Better Short-term Outcome 
for Mediastinal Tumor Surgery. Ann Thorac Surg 2019;108:1045-51.

14. Elkhouly A, Pompeo E. Nonintubated Subxiphoid Bilateral Redo Lung Volume Reduction Surgery. Ann Thorac Surg 2018;106:e277-9.

15. Pompeo E, Rogliani P, Atinkaya C, et al. Nonintubated surgical biopsy of undetermined interstitial lung disease: a multicentre outcome analysis. Interact Cardiovasc Thorac Surg 2019;28:744-50.

16. Gonzalez-Rivas D, Bonome C, Fieira E, et al. Nonintubated video-assisted thoracoscopic lung resections: the future of thoracic surgery? Eur J Cardiothorac Surg 2016;49:721-31.

17. Jiang L, Liu J, Gonzalez-Rivas D, et al. Thoracoscopic surgery for tracheal and carinal resection and reconstruction under spontaneous ventilation. J Thorac Cardiovasc Surg 2018;155:2746-54.

18. Li S, Jiang L, Ang KL, et al. New tubeless video-assisted thoracoscopic surgery for small pulmonary nodules. Eur J Cardiothorac Surg 2017;51:689-93.

19. Prisciandaro E, Bertolaccini L, Sedda G, et al. Nonintubated thoracoscopic lobectomies for lung cancer: an exploratory systematic review and meta-analysis. Interact Cardiovasc Thorac Surg 2020;31:499-506.

20. Cui F, Liu J, Li S, et al. Tubeless video-assisted thoracoscopic surgery (VATS) under non-intubated, intravenous anesthesia with spontaneous ventilation and no placement of chest tube postoperatively. J Thorac Dis 2016;8:2226-32.

21. Petersen RH, Holbek BL, Hansen HJ, et al. Video-assisted thoracoscopic surgery-taking a step into the future. Eur J Cardiothorac Surg 2017;51:694-5.

22. MacMahon H, Naidich DP, Goo JM, et al. Guidelines for Management of Incidental Pulmonary Nodules Detected on CT Images: From the Fleischner Society 2017. Radiology 2017;284:228-43.

23. Kim YW, Lee CT. Optimal management of pulmonary ground-glass opacity nodules. Transl Lung Cancer Res 2019;8:S418-24.

24. Callister MEJ, Baldwin DR, Akram AR on behalf of the British Thoracic Society Standards of Care Committee, et al. British Thoracic Society guidelines for the investigation and management of pulmonary nodules: accredited by NICE. Thorax 2015;70:ii1-ii54.

25. Chen PH, Chang KM, Tseng WC, et al. Invasiveness and surgical timing evaluation by clinical features of groundglass opacity nodules in lung cancers. Thorac Cancer 2019;10:2133-41.
26. Lococo F, Cusumano G, De Filippis AF, et al. Current Practices in the Management of Pulmonary Ground-Glass Opacities: A Survey of SICT Members. Ann Thorac Surg 2018;106:1504-11.

27. Yao J, He Z, Chen Y, et al. Acupuncture and weight loss in Asians: A PRISMA-compliant systematic review and metaanalysis. Medicine (Baltimore) 2019;98:e16815.

28. Zhang YL, Zhou C, Li XF, et al. Beinaglutide showed significant weight-loss benefit and effective glycaemic control for the treatment of type 2 diabetes in a realworld setting: a 3 -month, multicentre, observational, retrospective, open-label study. Obes Sci Pract 2019;5:366-75.

29. Coley EL, Irons JF. Non-intubated anesthetic techniques for thoracic surgery. Video-assist Thorac Surg 2017;2:69.

30. Wang ML, Hung MH, Chen JS, et al. Nasal high-flow oxygen therapy improves arterial oxygenation during onelung ventilation in non-intubated thoracoscopic surgery. Eur J Cardiothorac Surg 2018;53:1001-6.

31. Bratzler DW, Houck PM; Surgical Infection Prevention Guidelines Writers Workgroup, et al. Antimicrobial prophylaxis for surgery: an advisory statement from the National Surgical Infection Prevention Project. Clin Infect Dis 2004;38:1706-15.

32. ASHP Therapeutic Guidelines on Antimicrobial Prophylaxis in Surgery. American Society of HealthSystem Pharmacists. Am J Health Syst Pharm 1999;56:1839-88.

33. Price DD, McGrath PA, Rafii A, et al. The validation of visual analogue scales as ratio scale measures for chronic and experimental pain. Pain 1983;17:45-56.

34. Wewers ME, Lowe NK. A critical review of visual analogue scales in the measurement of clinical phenomena. Res Nurs Health 1990;13:227-36.

35. Myles PS, Myles DB, Galagher W, et al. Measuring acute postoperative pain using the visual analog scale: the minimal clinically important difference and patient acceptable symptom state. Br J Anaesth 2017;118:424-9.

36. Peng G, Liu M, Luo Q, et al. Spontaneous ventilation anesthesia combined with uniportal and tubeless thoracoscopic lung biopsy in selected patients with interstitial lung diseases. J Thorac Dis 2017;9:4494-501.

37. Hung WT, Hung MH, Wang ML, et al. Nonintubated Thoracoscopic Surgery for Lung Tumor: Seven Years' Experience With 1,025 Patients. Ann Thorac Surg 2019;107:1607-12.

38. Pompeo E, Sorge R, Akopov A, et al. Non-intubated thoracic surgery-A survey from the European Society of 
Thoracic Surgeons. Ann Transl Med 2015;3:37.

39. Liu CY, Hsu PK, Chien HC, et al. Tubeless single-port thoracoscopic sublobar resection: indication and safety. J Thorac Dis 2018;10:3729-37.

40. Lirio F, Galvez C, Bolufer S, et al. Tubeless major pulmonary resections. J Thorac Dis 2018;10:S2664-70.

41. Suzuki K, Koike T, Asakawa T, et al. A prospective radiological study of thin-section computed tomography to predict pathological noninvasiveness in peripheral clinical IA lung cancer (Japan Clinical Oncology Group 0201). J

Cite this article as: $\mathrm{He} \mathrm{J}$, Liang $\mathrm{H}$, Wang W, Akopov A, Aiolfi A, Ang KL, Bertolaccini L, Cai K, Cao Q, Chen B, Chen C, Chen C, Chen D, Chen F, Chen J, Chen L, Chen M, Chen Y, Chen Z, Cheng C, Cui D, Cui F, Dai T, Dong Q, Ferrari PA, Flores RM, Fu J, Funaki S, Froudarakis ME, Gan X, Geng M, Guo J, Guo Q, Han Y, He J, He K, Hirai K, Hu J, Hu S, Huang J, Huang J, Jiang W, Kim KS, Kiss G, Kong F, Lan L, Leng X, Li B, Li G, Li H, Li H, Li H, Li J, Li X, Li S, Li Y, Li Z, Liang Y, Liang L, Liang W, Liao Y, Lin W, Lin X, Liu H, Liu H, Liu J, Liu J, Liu X, Liu Z, Lu X, Luo Q, Mao N, Pan Q, Pang D, Peng J, Peng J, Pompeo E, Qian R, Qiao K, Redwan B, Sang Z, Shao W, Shen J, Shen W, Sung SW, Tang W, Wang T, Wang G, Wang H, Wang H, Wang J, Wang W, Wang Y, Wang Z, Wei L, Wei W, Wu H, Wu J, Xia Z, Xu C, Xu E, Xu H, Xu N, Xu Q, Xu R, Xu S, Yang C, Yang H, Yang S, Yi J, Zhang G, Zhang H, Zhang J, Zhang M, Zhang X, Zhang Y, Zhang Z, Zhang Z, Zhao H, Zhao J, Zhao X, Zhou J, Zhou Y, Zhu C, Zhu S, Zhu X, Cui J, Yan Y, Chen KN. Tubeless video-assisted thoracic surgery for pulmonary ground-glass nodules: expert consensus and protocol (Guangzhou). Transl Lung Cancer Res 2021;10(8):3503-3519. doi: 10.21037/tlcr-21-663
Thorac Oncol 2011;6:751-6.

42. Suzuki K, Asamura H, Kusumoto M, et al. "Early" peripheral lung cancer: prognostic significance of ground glass opacity on thin-section computed tomographic scan. Ann Thorac Surg 2002;74:1635-9.

43. Cho JH, Choi YS, Kim J, et al. Long-term outcomes of wedge resection for pulmonary ground-glass opacity nodules. Ann Thorac Surg 2015;99:218-22.

(English Language Editor: J. Gray) 